\title{
Frequency of Transducer-like Enhancer of Split 1 Immunohistochemical Expression in Synovial Sarcoma: An Institution-based Cross-sectional Study
}

Madiha B. Oureshi ${ }^{1}$, Nasir Uddin ${ }^{1}$, Muhammad Usman Tariq ${ }^{1}$, Ahmed Raheem ${ }^{1}$, Shahid Pervez ${ }^{2,} 3$

1. Pathology and Laboratory Medicine, Aga Khan University Hospital, Karachi, PAK 2. Pathology and Laboratory Medicine, Aga Khan University Hospital, Karachi, PAK 3. Pathology and Laboratory Medicine, Intermountain Healthcare, Salt Lake City, USA

Corresponding author: Madiha B. Qureshi, madihabilal.qureshi@aku.edu

\section{Abstract \\ Background}

Soft-tissue sarcomas comprise a diverse group of sarcomas with characteristic histologic features. However, histology alone is not adequate for a definitive diagnosis for many tumors. In such cases, immunohistochemistry (IHC) plays a key role in determining the line of differentiation and exact characterization. Transducer-like enhancer of split 1 (TLE1) has been recently described as a novel marker for synovial sarcoma (SS). Its high sensitivity and specificity make it a potential marker that distinguishes SS from histologic mimics such as malignant peripheral nerve sheath tumor (MPNST), Ewing's sarcoma (ES), and fibrosarcomatous dermatofibrosarcoma protuberans (FS-DFSP). The objective of our study was to assess the frequency of TLE1 immunohistochemical expression on SS cases of various subtypes.

\section{Methods}

This cross-sectional study was conducted at the Department of Histopathology, Aga Khan University, Karachi, Pakistan from February 3, 2018 to February 10, 2019. Tissue samples of 89 SS cases were selected for this study. Tumor sections were stained with hematoxylin and eosin (H\&E), cytokeratin AEI/AE3 (CKAE1/AE3), epithelial membrane antigen (EMA), and TLE1 immunohistochemical stain. TLE1 expression was assessed based on the Remmele scoring system.

\section{Results}

Tissue samples of 89 SS cases were processed for the study. Mean $( \pm$ ) standard deviation (SD) of age was 25 $( \pm 7.36)$ years. Male:female ratio was $1.1: 1$. Of the 89 SS cases, 42 (47.2\%) were monophasic, six (6.7\%) were biphasic, and 41 (46.1\%) were poorly differentiated. All the 89 cases showed positivity for TLE1 immunostain: 86 (96.6\%) cases showed strong positivity, one (1.1\%) case showed moderate expression, and

Received 09/06/2019

Review began 09/13/2019

Review ended 10/20/2019

Published 12/11/2019

๑) Copyright 2019

Qureshi et al. This is an open access article distributed under the terms of the Creative Commons Attribution License CC-BY 3.0., which permits unrestricted use, distribution, and reproduction in any medium, provided the original author and source are credited. two (2.2\%) showed weak positivity.

\section{Conclusion}

This study shows that TLE1 is a highly sensitive immunostain for SS irrespective of the histologic type. However, it may show weak-to-moderate staining in poorly differentiated types. No statistically significant association was seen with respect to age group, gender, or type of SS.

Categories: Pathology, General Surgery, Oncology

Keywords: synovial sarcoma, monophasic synovial sarcoma, poorly differentiated synovial sarcoma, tle1

\section{Introduction}

Synovial sarcoma (SS) is the fourth most common sarcoma, comprising $10 \%$ of all soft-tissue sarcomas [1-3]. It has a relatively higher occurrence in the 15-49 age group in Karachi [4]. Most of these tumors (80\%) arise in the deep intramuscular soft tissues of extremities, especially around the knee, ankle, feet, and hand. The other commonly affected sites include the inguinal region, abdominal wall, and head and neck. Virtually every site has been reported including nerve, heart, lung, prostate, kidney, etc. [5-6].

Three histologic types of SS are known: monophasic (50-60\%), which consists of monomorphic spindleshaped cells arranged in sheets or fascicles and rare mitosis with differentials of malignant peripheral nerve sheath tumor (MPNST), fibrosarcomatous dermatofibrosarcoma protuberans (FS-DFSP), etc.; biphasic (20$30 \%$ ), which consists of both spindle and epithelial components; and poorly differentiated SS (10-15\%), which consists of diffuse sheets of small round blue cells with nuclear atypia, conspicuous nucleoli, and high mitotic rate with close differential of Ewing's sarcoma (ES) [7-10]. 
The tumor is characterized by a $\mathrm{t}(\mathrm{X} ; 18)$ balanced translocation, which results in a fusion of the SSX gene present on chromosome $X$ to the SYT gene on chromosome 18 [1-11]. The SYT-SSX fusion oncoprotein causes transcriptional dysregulation by repression of tumor-suppressor genes [12,13]. Hence, the gold standard for SS diagnosis is fluorescence in situ hybridization (FISH), reverse transcriptase-polymerase chain reaction (RT-PCR), or cytogenetics. However, unfortunately, in a developing country like Pakistan, the lack of well-equipped laboratories, skilled personnel, and financial constraints limit their use [1,2,14].

Traditionally, a panel of immunohistochemical stains (none of which is specific) including epithelial membrane antigen (EMA), cytokeratins (CK AE1/AE3, CK7, CK19), cluster of differentiation (CD99, CD34), B cell lymphoma 2 (BCL-2), and vimentin are used for SS diagnosis $[1,13,15]$. Recent studies have shown Transducer-like enhancer of split 1 (TLE1) to be more sensitive and specific than all the other biomarkers in diagnosing and differentiating SS from histologic mimics [1,3]. TLE1 is a member of the Groucho/TLE gene family and encodes a transcriptional protein necessary for hematopoiesis and cellular differentiation $[16,17]$. TLE1 protein is also involved in the Wnt/ $\beta$-catenin signaling pathway associated with SS, where it replaces TLE1-TCT/LEF complexes that repress transcription [18-20]. Genetic studies have identified TLE1 as a robust biomarker for differentiating SS from its histologic identicals [21-23].

Several studies worldwide have demonstrated variable sensitivities ranging from $75-95.2 \%$ for TLE1 as an immunomarker for SS, with two of them concluding it to be more sensitive for the poorly differentiated subtype $[2,3,13,18]$.

No prior research work has been done in our part of the world to assess the diagnostic utility of TLE1 in SS. Also, relative TLE1 expression in different subtypes needs to be thoroughly studied for differentiating these subtypes from their histologic mimics on an immunohistochemistry (IHC) basis. This study will evaluate the expression of TLE1 in a cohort of SS cases including all subtypes in routine diagnostic practice.

\section{Materials And Methods}

The study was performed at the Section of Histopathology, Department of Pathology and Laboratory Medicine, Aga Khan University Hospital, Karachi. All diagnosed SS cases received as incisional tumor biopsies or surgical resection specimens were included in the study. These specimens included both genders of age ranging between 15-35 years, and with tumors from any site of the body. Consent was taken from the parents of patients in the pediatric age group. The diagnosis was given based on classic morphology and positive IHC of immunostains cytokeratin AE1/AE3 and EMA. Poorly fixed or autolyzed specimen (specimen not received in $10 \%$ buffered formalin), tru-cut biopsy (TCB) specimens, cases with the diagnosis that says "suggestive of", and cases diagnosed with other differentials were excluded. Testing was performed on biopsied tissues. Formalin-fixed, paraffin-embedded tissue sections with a thickness of four micrometers were deparaffinized using xylene, rehydrated via dehydrated alcohols, and treated with $10 \mathrm{mM}$ citrate buffer at pH 6.0 at 450 watts in the microwave for 20 minutes to retrieve the antigen. Next, the sections were placed under tap water and the application of hydrogen peroxide blocker was done for up to five minutes in order to block endogenous enzyme peroxidase activity. Distilled water was used for washing slides and the primary antibody, Cell Marque TLE1 monoclonal antibody (Sigma-Aldrich Co. LLC, St. Louis, Missouri), was administered at a dilution of 1:100 for 30 minutes at room temperature. After that, the slides were washed with wash buffer, and polymer labeled with enzyme HRP was applied for 30 minutes at room temperature. Immunostaining was performed using Dako Envision FLEX method with threediaminobenzidine (DAB) as a chromogen for 10 minutes. Hematoxylin solution was used for counterstaining. Positive known control, as well as the negative control (wash buffer to take the place of the first antibody), was included and run with each batch of IHC staining. Finally, slides were dried in an oven and mounted with mounting media (DPX).

Dark brown nuclear staining was the only result interpreted as positive. The cases were initially analyzed and scored by the principal investigator, and findings were reviewed and confirmed by a consultant histopathologist. Variables including age, sex, patient identification, specimen type, diagnosis (SS type), and immunohistochemical marker positivity were obtained on a predesigned proforma. For IHC staining analysis, the stained slides were examined under a light microscope to look for proportion and intensity of staining according to the Remmele score (0-12) as follows [3]: Remmele score $=$ intensity of immunoreactivity $\times$ percentage of the stained tumor cells.

Intensity of immunoreactivity for the Remmele Score was assessed as follows: zero for no staining, one for weak staining (faint light-brown staining), two for moderate/intermediate staining (dark-brown nuclear staining of intensity less than that of positive control), and three for strong staining (dark-brown nuclear staining with similar intensity of positive control). Percentage of positively stained cells was assessed as follows: zero for no staining, one for $<10 \%$ of the cells staining, two for $11-50 \%$ of the cells staining, three for $51-80 \%$ of the cells staining, and four for $81-100 \%$ of the cells staining. The cumulative score was interpreted as follows: five to twelve = high score, three to four = moderate score, one to two = weak score, and zero = negative score. High and moderate scores were considered as positive TLE1 staining, whereas weak score was considered negative. 


\section{Cureus}

(IBM, Armonk, NY). Descriptive statistics were determined. Mean $( \pm)$ standard deviation (SD) was assessed for quantitative variables such as the age of the patient and the Remmele score. Frequency and percentages were also calculated for qualitative variables such as gender distribution, SS type (monophasic, biphasic, and poorly differentiated), and TLE1 IHC staining (yes/no). Effect modifier was kept under control through stratification with regards to age, gender, and type of SS to measure the outcome on TLE1. A chi-square test was utilized, and a probability value (p) of $\leqslant 0.05$ was considered significant.

\section{Results}

Tissue samples of 89 SS cases were selected for the study. Mean $( \pm)$ SD of age was $25( \pm 7.36)$ years. Male:female ratio was 1.1:1. Of the 89 patients, 47 (53\%) were female and 42 (47\%) were male.

Of all the 89 SS cases, 42 (47.2\%) were monophasic, six (6.7\%) were biphasic, and 41 (46.1\%) were of poorly differentiated type. The most common site of tumor occurrence was thigh (13 cases), followed by leg (12), and arm (nine). Other sites included the knee, foot, forearm, neck, and buttocks, etc. (Table 1).

\begin{tabular}{|c|c|c|}
\hline Site & Frequency (f) & Percentage (\%) \\
\hline Posterior aural & 1 & 1.10 \\
\hline Lymph node & 1 & 1.10 \\
\hline Unknown/not specified & 2 & 2.20 \\
\hline Leg & 12 & 13.50 \\
\hline Neck & 5 & 5.60 \\
\hline Face & 3 & 3.40 \\
\hline Arm & 9 & 10.10 \\
\hline Temporal/extradural & 2 & 2.20 \\
\hline Chest & 4 & 4.50 \\
\hline Knee & 8 & 9.00 \\
\hline Hand & 2 & 2.20 \\
\hline Forearm & 6 & 6.70 \\
\hline Kidney & 1 & 1.10 \\
\hline Popliteal & 2 & 2.20 \\
\hline Groin & 1 & 1.10 \\
\hline Foot & 8 & 9.00 \\
\hline Buttock & 4 & 4.50 \\
\hline Retroperitoneal & 1 & 1.10 \\
\hline Vocal cord & 1 & 1.10 \\
\hline Pelvic & 1 & 1.10 \\
\hline Pleura & 1 & 1.10 \\
\hline Thigh & 13 & 14.60 \\
\hline Lung & 1 & 1.10 \\
\hline Total & 89 & 100 \\
\hline
\end{tabular}

TABLE 1: Site distribution of tumors

The percentage of immunohistochemically positive cells was noted for each case (Table 2). Intensity of immunoreactivity for TLE1 immunostain was recorded for each case (Table 2 and Figure 1). The Remmele score was calculated as described and was found to be equal to and in between two and twelve. Hence, all 


\section{Cureus}

the SS cases were interpreted as positive for TLE1 immunostain.

\begin{tabular}{|c|c|c|}
\hline TLE1 positive cells & Frequency (f) & Percentage (\%) \\
\hline$<10 \%$ of cells staining & 1 & 1.10 \\
\hline $11-50 \%$ of cells staining & 6 & 6.70 \\
\hline $51-80 \%$ of cells staining & 14 & 15.70 \\
\hline $81-100 \%$ of cells staining & 68 & $/ 6.40$ \\
\hline Total & 89 & 100 \\
\hline \multicolumn{3}{|l|}{ Intensity of TLE1 staining } \\
\hline Weak positive & 2 & 2.20 \\
\hline Intermediate positive & 1 & 1.10 \\
\hline Strong positive & 86 & 96.60 \\
\hline Total & 89 & 100 \\
\hline
\end{tabular}

TABLE 2: TLE1 immunohistochemical expression

TLE1: transducer-like-enhancer of split 1

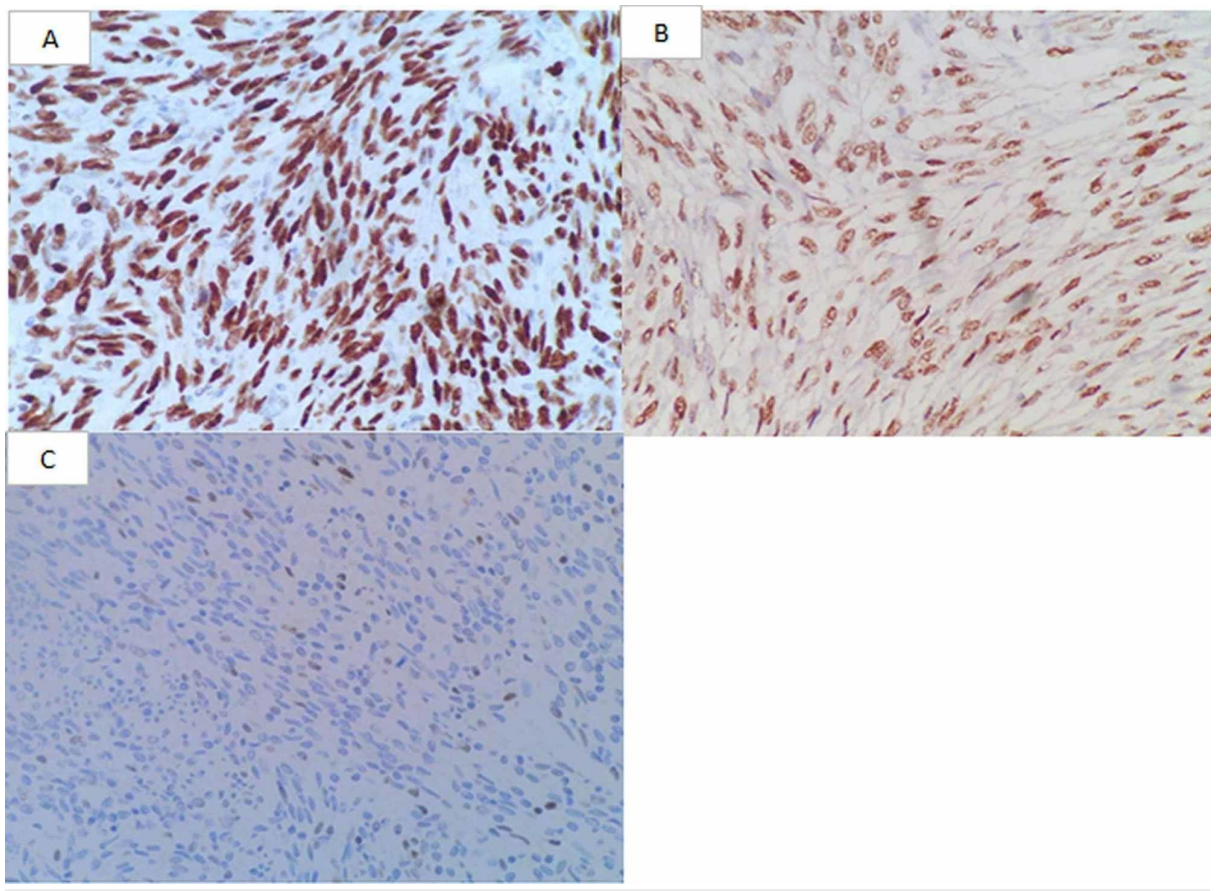

FIGURE 1: TLE1 immunostaining

(A) Strong nuclear TLE1 immunostaining in monophasic type synovial sarcoma, H\&E, 20x

(B) Moderate/intermediate nuclear TLE1 immunostaining in monophasic type synovial sarcoma, H\&E, 20x

(C) Weak nuclear TLE1 immunostaining in monophasic type synovial sarcoma, H\&E, 20x

Since all the cases showed TLE1 immunopositivity, effect modifier was kept under control via stratification with regards to age, gender, and type of SS to measure the outcome on TLE1 as strong positive, 
moderate/intermediate positive, and weak positive. Three age groups were thus made: 15-20 years, 21-30 years, and 31-35 years.

Out of the 89 cases, 32 (36\%) were aged 31-35 years, 31 (34.8\%) were aged 21 -30 years, and 26 (29.2\%) were aged 15-20 years. TLE1 interpretation with respect to age groups, gender, and type of SS is shown (Table 3).

\begin{tabular}{|c|c|c|c|c|c|}
\hline \multirow{2}{*}{ Study variables } & \multicolumn{4}{|c|}{ TLE1 interpretation } & \multirow{3}{*}{ P-value } \\
\hline & Negative & Weak positive & Intermediate positive & Strong positive & \\
\hline \multicolumn{5}{|l|}{ Age groups } & \\
\hline $15-20$ years & $0(0 \%)$ & $0(0 \%)$ & $0(0 \%)$ & $26(29.2 \%)$ & \multirow{3}{*}{0.615} \\
\hline $21-30$ years & $0(0 \%)$ & $1(1.1 \%)$ & $0(0 \%)$ & $30(33.7 \%)$ & \\
\hline $31-35$ years & $0(0 \%)$ & $1(1.1 \%)$ & $1(1.1 \%)$ & 30 (33.7\%) & \\
\hline \multicolumn{6}{|l|}{ Gender } \\
\hline Male & $0(0 \%)$ & $2(2.2 \%)$ & $0(0 \%)$ & 40 (44.9\%) & \multirow{3}{*}{0.207} \\
\hline Female & $0(0 \%)$ & $0(0 \%)$ & $1(1.1 \%)$ & $46(51.7 \%)$ & \\
\hline \multicolumn{5}{|l|}{ Synovial sarcoma type } & \\
\hline Monophasic & $0(0 \%)$ & $0(0 \%)$ & $0(0 \%)$ & $42(47.2 \%)$ & \multirow{3}{*}{0.458} \\
\hline Biphasic & $0(0 \%)$ & $0(0 \%)$ & $0(0 \%)$ & $6(6.7 \%)$ & \\
\hline Poorly differentiated & $0(0 \%)$ & $2(2.2 \%)$ & $1(1.1 \%)$ & $38(42.7 \%)$ & \\
\hline
\end{tabular}

TABLE 3: TLE1 interpretation based on age, gender, and synovial sarcoma type

TLE1: transducer-like-enhancer of split 1

\section{Discussion}

It is quite challenging to distinguish SS from histologic mimics on morphology alone [16]. The immunostains currently available including CK AE1/AE, EMA, the cluster of differentiation (CD99, CD34), B-cell lymphoma 2 (BCL-2), and vimentin are less sensitive and specific [12,18]. Genetic analysis has proved TLE1, a gene that exhibits increased expression in SS, to be a remarkable biomarker [17]. There have been several works of research in different parts of the world assessing TLE1 expression in SS with differential expression in occasional studies only. No data have been published in Pakistan on the relevant subject. In this study, the age range is comparable to studies done internationally. The sample size of the study included both incisional tumor biopsies and surgical resection specimens.

In comparison to a study by Knosel et. al., which showed 96\% positivity for TLE1, this study has shown $100 \%$ TLE1 expression [3]. Strong and moderate TLE1 staining was observed in $97.7 \%$ of the cases, whereas weak expression was seen in $2.2 \%$, in comparison to the $75 \%$ strong and moderate positive and $21 \%$ weak positive cases in the study by Knosel et. al. Another study done by Jagdis et al. showed strong TLE1 positivity in all cases of poorly differentiated SS, which is different from the findings of this study in which only $3.3 \%$ of poorly differentiated SS cases showed weak and moderate staining [2]. In the one case of biphasic SS in the study by Jagdis et al., TLE1 was strong positive in both spindle and epithelial cells and this finding is similar to our study.

In another study by Foo et al., $82 \%$ of SS cases displayed positivity for TLE1, including $78 \%$ biphasic, $79 \%$ monophasic, and $91 \%$ poorly differentiated types [16]. Similarly, in a study by Chuang et al., $78-86 \%$ of SS cases showed nuclear TLE1 expression [1]. This again is in contrast with the current study, which showed 100\% TLE1 expression.

Limitations that are conceivable in this study include a lack of financial resources for performing $\mathrm{t}(\mathrm{X} ; 18)$ translocation for each case. A comparison of TLE1 expression in other sarcomas would provide much greater insight into establishing the specificity of this robust marker. As far as the findings of this study are concerned, TLE1 is an extremely sensitive immunomarker for SS, irrespective of the type. The greater the staining intensity, the higher the sensitivity. 


\section{Conclusions}

This study shows that TLE1 has a high sensitivity for SS as an immunomarker irrespective of the histologic type. However, it may show weak-to- moderate staining in poorly differentiated types of SS. No statistically significant association was seen with respect to age group, gender, or SS type.

\section{Additional Information \\ Disclosures}

Human subjects: Consent was obtained by all participants in this study. AKU-RRG-Z014-03 issued approval Z014-03. Thank you for your application for exemption from ethical approval regarding the above mentioned study. Your study was reviewed and approved as exemption. Please ensure that the study is performed as per protocol following all AKU standards. You may proceed with the study. Thank you. Sincerely. Animal subjects: All authors have confirmed that this study did not involve animal subjects or tissue. Conflicts of interest: In compliance with the ICMJE uniform disclosure form, all authors declare the following: Payment/services info: All authors have declared that no financial support was received from any organization for the submitted work. Financial relationships: All authors have declared that they have no financial relationships at present or within the previous three years with any organizations that might have an interest in the submitted work. Other relationships: All authors have declared that there are no other relationships or activities that could appear to have influenced the submitted work.

\section{References}

1. Chuang HC, Hsu SC, Huang CG, Hsueh S, Ng KF, Chen TC: Reappraisal of TLE-1 immunohistochemical staining and molecular detection of SS18-SSX fusion transcripts for synovial sarcoma. Pathol Int. 2013, 63:573-80. 10.1111/pin.12113

2. Jagdis A, Rubin BP, Tubbs RR, Pacheco M, Nielsen TO: Prospective evaluation of TLE1 as a diagnostic immunohistochemical marker in synovial sarcoma. Am J Surg Pathol. 2009, 33:1743-51. 10.1097/PAS.0b013e3181b7ed36

3. Knosel T, Heretsch S, Altendorf-Hofmann A, et al.: TLE1 is a robust diagnostic biomarker for synovial sarcomas and correlates with $\mathrm{t}(\mathrm{X} ; 18)$ : analysis of 319 cases. Eur J Cancer. 2010, 46:1170-6. 10.1016/j.ejca.2010.01.032

4. Bhurgri Y, Bhurgri H, Pervez S, et al.: Epidemiology of soft tissue sarcomas in Karachi South, Pakistan (1995-7). Asian Pac J Cancer Prev. 2008, 9:709-14.

5. Cadman NL, Soule EH, Kelly PJ: Synovial sarcoma; an analysis of 134 tumors . Cancer. 1965, 18:613-27. 10.1002/1097-0142(196505)18:5<613::aid-cncr2820180510>3.0.co;2-V

6. Thway K, Fisher C: Synovial sarcoma: defining features and diagnostic evolution . Ann Diagn Pathol. 2014, 18:369-80. 10.1016/j.anndiagpath.2014.09.002

7. Fisher C: Synovial sarcoma. Ann Diagn Pathol. 1998, 2:401-21. 10.1016/S1092-9134(98)80042-7

8. Tanaka K, Ozaki T: New TNM classification (AJCC eighth edition) of bone and soft tissue sarcomas: JCOG Bone and Soft Tissue Tumor Study Group. Jpn J Clin Oncol. 2019, 49:103-7. 10.1093/jjco/hyy157

9. Ferrari A, Gronchi A, Casanova M, et al.: Synovial sarcoma: a retrospective analysis of 271 patients of all ages treated at a single institution. Cancer. 2004, 101:627-34. 10.1002/cncr.20386

10. Vlenterie M, Litière S, Rizzo E, et al.: Outcome of chemotherapy in advanced synovial sarcoma patients: review of 15 clinical trials from the European Organisation for Research and Treatment of Cancer Soft Tissue and Bone Sarcoma Group; setting a new landmark for studies in this entity. Eur J Cancer. 2016, 58:62-72. 10.1016/j.ejca.2016.02.002

11. Vlenterie M, Hillebrandt-Roeffen MH, Flucke UE, et al.: Next generation sequencing in synovial sarcoma reveals novel gene mutations. Oncotarget. 2015, 6:34680-90. Accessed: October 23, 2019: 10.18632/oncotarget.5786

12. Terry J, Saito T, Subramanian S, et al.: TLE1 as a diagnostic immunohistochemical marker for synovial sarcoma emerging from gene expression profiling studies . Am J Surg Pathol. 2007, 31:240-6. 10.1097/01.pas.0000213330.71745.39

13. Rekhi B, Basak R, Desai SB, Jambhekar NA: Immunohistochemical validation of TLE1, a novel marker, for synovial sarcomas. Indian J Med Res. 2012, 136:766-75.

14. Jain S, Xu R, Prieto VG, Lee P: Molecular classification of soft tissue sarcomas and its clinical applications . Int J Clin Exp Pathol. 2010, 3:416-28. Accessed: October 23, 2019: https://www.ncbi.nlm.nih.gov/pubmed/20490332.

15. Fisher C: Immunohistochemistry in diagnosis of soft tissue tumours . Histopathology. 2011, 58:1001-12. 10.1111/j.1365-2559.2010.03707.x

16. Foo WC, Cruise MW, Wick MR, Hornick JL: Immunohistochemical staining for TLE1 distinguishes synovial sarcoma from histologic mimics. Am J Clin Pathol. 2011, 135:839-44. 10.1309/AJCP45SSNAOPXYXU

17. Ladanyi M: Fusions of the SYT and SSX genes in synovial sarcoma . Oncogene. 2001, 20:5755-62. 10.1038/sj.onc.1204601

18. Kosemehmetoglu K, Vrana JA, Folpe AL: TLE1 expression is not specific for synovial sarcoma: a whole section study of 163 soft tissue and bone neoplasms. Mod Pathol. 2009, 22:872-8. 10.1038/modpathol.2009.47

19. Haldar M, Randall RL, Capecchi MR: Synovial sarcoma: from genetics to genetic-based animal modeling . Clin Orthop Relat Res. 2008, 466:2156-67. 10.1007/s11999-008-0340-2

20. Pelmus M, Guillou L, Hostein I, Sierankowski G, Lussan C, Coindre JM: Monophasic fibrous and poorly differentiated synovial sarcoma: immunohistochemical reassessment of $60 \mathrm{t}$ (X; 18) (SYT-SSX)-positive cases. Am J Surg Pathol. 2002, 26:1434-40. 10.1097/00000478-200211000-00005 


\section{Cureus}

21. He R, Patel RM, Alkan S, et al.: Immunostaining for SYT protein discriminates synovial sarcoma from other soft tissue tumors: analysis of 146 cases . Mod Pathol. 2007, 20:522-8. 10.1038/modpathol.3800766

22. Jo VY, Fletcher CD: WHO classification of soft tissue tumours: an update based on the 2013 (4th) edition . Pathol. 2014, 46:95-104. 10.1097/PAT.0000000000000050

23. Lagarde P, Przybyl J, Brulard C, et al.: Chromosome instability accounts for reverse metastatic outcomes of pediatric and adult synovial sarcomas. J Clin Oncol. 2013, 31:608-15. 10.1200/JCO.2012.46.0147 